\title{
Analysis of Pulsatile Magnetohydrodynamic (MHD) Third Grade Blood Flow in a Stenosed Artery
}

\author{
L. N. Ikpakyegh ${ }^{1}$, G. T. Okedayo ${ }^{2}$, T. Aboiyar ${ }^{1}$, E. S. Onah ${ }^{1}$ \\ ${ }^{1}$ Department of Mathematics/Statistics/Computer Science, University of Agriculture Makurdi, Benue State, Nigeria \\ ${ }^{2}$ Department of Mathematical Sciences, Ondo State University of Science and Technology Okitipupa, Ondo State, Nigeria \\ Email: lewisnyitor@yahoo.com, okedayo@yahoo.com,t_aboiyar@yahoo.co.uk
}

How to cite this paper: Ikpakyegh, L.N., Okedayo, G.T., Aboiyar, T. and Onah, E.S. (2018) Analysis of Pulsatile Magnetohydrodynamic (MHD) Third Grade Blood Flow in a Stenosed Artery. American Journal of Computational Mathematics, 8, 78-95. https://doi.org/10.4236/ajcm.2018.81007

Received: January 9, 2018

Accepted: March 25, 2018

Published: March 28, 2018

Copyright $\odot 2018$ by authors and Scientific Research Publishing Inc. This work is licensed under the Creative Commons Attribution International License (CC BY 4.0).

http://creativecommons.org/licenses/by/4.0/

\begin{abstract}
In this research, we modeled MHD third grade blood flow in a stenosed artery. The blood viscosity and the density have been modeled into the shear thinning/thickening parameters, the most important rheological properties of blood. We used regular perturbation method and obtained the flow characteristics such as the flow velocity, the volume flow rate, the shear stress and the resistance to the flow considering a single layered stenosed artery. The results however showed that there is significant increase in volume flow rate and the velocity with increase in the magnetic field intensity $H$ and the shear thinning $\Lambda$ and reduces with increase in the shear thickening $\Omega$.
\end{abstract}

\section{Keywords}

Third Grade Fluid, Magnetohydrodynamics (MHD), Shear

Thinning/Thickening

\section{Introduction}

The term viscosity (the ability of fluids to resist gradual deformation by shear or tensile stresses) is commonly used in fluid mechanics to characterize the shear properties of a fluid. It cannot be enough to describe non-Newtonian fluids like blood. Blood is a body fluid in humans and other animals, which delivers necessary substances such as nutrients and oxygen to the cells through some body canals called arteries and transports metabolic waste products away from those same cells through veins. So far, blood is best studied through several other rheological properties that relate stress and strain rate tensors under many different flow conditions such as oscillatory shear or extensional flow which is 
measured using different devices or rheometers. These properties are better studied using tensor-valued constitutive equations, which are common in the field of continuum mechanics. According to [1], blood behaves differently when flowing in large vessels, in which Newtonian behavior is expected and in medium and small vessels where non-Newtonian effects appear.

Arteries transport oxygenated blood to different tissues and organs of the body. This is essential for metabolic activities and sustaining life functions. Any narrowing of an artery will hamper the flow of blood and depending on the extent of the narrowing, known as stenosis, the artery may be partially or completely occluded (blocked) [2]. Narrowing of an artery may not cause obvious signs and symptoms in the initial stages. If sufficient oxygenated blood is able to reach the target organs and tissues, the narrowing may go unnoticed for long periods of time. As the condition progresses, the flow of blood will be hampered to a degree that affects the oxygen supply to the target tissue. It is usually at this stage that the first signs and symptoms become evident [3]. A sudden occlusion of an artery (blocked artery) can lead to a host of life threatening illnesses, including a myocardial infarction (heart attack), stroke or pulmonary embolism. However, slow and progressive narrowing may remain unnoticed for weeks, months or years [3].

A non-Newtonian fluid is one whose flow curve (shear stress versus shear rate) is nonlinear or does not pass through the origin, i.e. where the apparent viscosity, shear stress divided by shear rate, is not constant at a given temperature and pressure but is dependent on flow conditions such as flow geometry, shear rate, etc. and sometimes even on the kinematic history of the fluid element under consideration.

The study of non-Newtonian fluid flow is important due to the nonlinear fluid rheology which is of special interest and has practical applications in industry and engineering. Several fluid models have been suggested to disclose the nature of non-Newtonian fluids. Among them is the differential type. The differential type fluid model has a simplest subclass known as the second-grade fluid, which describes the normal stress differences but cannot predict the shear thinning/thickening phenomena. However, the third-grade fluid model is capable of predicting both the normal stresses and the shear thinning/thickening phenomena, the most important rheological properties of blood. In [4], the study of the steady flow of an incompressible, third-grade fluid in helical screw rheometer (HSR) was conducted by "unwrapping or flattening" the channel, lands, and the outside rotating barrel. In [5], the authors considered steady boundary layer axisymmetric flow of third-grade fluid over a continuously stretching cylinder in the presence of magnetic field. They used homotopy analysis method (HAM) to solve the differential equations.

The use of electromagnetic fields in the healing arts dates back as far as the fifteenth century, although the magnetic effects of "lodestone" were first described by the shepherd Magnes circa 1000 BCE in the region known today as 
Turkey [6]. In the $18^{\text {th }}$ century, Mesmer began treating hysteria and other disorders (today recognized to be psychosomatic in origin) with lodestones [6].

In the 1930s, it was found that haemoglobin has magnetic properties that are different depending on whether it is carrying oxygen or not. When the haemoglobin is not carrying oxygen it is more sensitive or paramagnetic than oxygenated blood [7]. Recently, however, interest in magnetic field therapy has revived, and a variety of products are available for treatment of humans.

The effects of applying a magnetic field to liquid water have been intensively studied since 1980. it has been shown that the water vaporization rate, an essential process for all biological processes, is significantly affected by the application of a static magnetic field in both air and oxygen environments [8]. Studies have also found that various aspects of liquid water structure, including the size of the water cluster change when exposed to a magnetic field [9]. Blood can be regarded as magnetic fluid, in which red blood cells are magnetic in nature. Liquid carriers in the blood contain the magnetic suspension of the particle [10]. In [11], the author studied the effect of magnetic field on blood samples. Blood was extracted from donors most of them were men with polycythemia (hyper-viscosity) disease. They considered $(10 \mathrm{ml})$ blood samples. Anti-clotting tubes were put under the influence of a steady magnetic field (MRI) (1.5 Tesla) for different exposure time. It was clearly observed that the viscosity of blood samples decreased with increasing the exposure time to magnetic fields. The decrease in viscosity was great for samples exposed for (1) minute and (15) minutes.

Although, the constitutive equations for third grade fluids have various complexities, many researchers have examined the flows of third-grade fluid under various aspects. We will take the study under a pulsatile condition and therefore begin by considering the flow of electrically conducting fluid (blood) in a stenosed artery.

The continuity and momentum equations are given as follows

$$
\begin{gathered}
\operatorname{div} \bar{u}=0 \\
\bar{\rho}_{T} \frac{\partial}{\partial \bar{t}} \bar{u}_{T}=-\frac{\partial}{\partial \overline{\mathrm{Z}}} \bar{P}-\frac{1}{r} \frac{\partial}{\partial \bar{r}}\left(\bar{r} \bar{\tau}_{T}\right)+\bar{\mu}_{0} M \frac{\partial}{\partial \overline{\mathrm{Z}}} \bar{H}
\end{gathered}
$$

Generally, for both Newtonian and non-Newtonian fluids, the Cauchy stress tensor is obtained by

$$
\tau=-P I+\sum_{j=1}^{n} S_{j}
$$

$S_{j}, j=1,2,3$ are called the stress tensors, $P$ is the pressure force due to fluid flow. The pressure force is directed against the flow direction, which is the axial direction to induce the fluid flow. For the third grade fluid we have $n=3$ and the first three tensors $S_{j}$ are given by

$$
\begin{gathered}
S_{1}=\bar{\mu} A_{1} \\
S_{2}=\alpha_{1} A_{2}+\alpha_{2} A_{1}^{2} \\
S_{3}=\beta_{1} A_{3}+\beta_{2}\left(A_{2} A_{1}+A_{1} A_{2}\right)+\beta_{3}\left(t r A_{1}^{2}\right) A_{1}
\end{gathered}
$$


where $\bar{\mu}$ is the coefficient of sheer viscosity and $\alpha_{i},(i=1,2), \beta_{i},(i=1,2,3)$ are material constants. $A_{n}$ are called Rivlin Ericksen tensors [12], and are defined by the recursion relation

$$
\begin{gathered}
A_{n}=\frac{D}{D t} A_{n-1}+A_{n-1}(\nabla \bar{u})+(\nabla \bar{u})^{\mathrm{T}} A_{n-1}, n>1 \\
A_{1}=(\nabla \bar{u})+(\nabla \bar{u})^{\mathrm{T}}
\end{gathered}
$$

When $\beta_{j}=0,(j=1,2,3)$, then, the above model reduces to second grade fluid model and if $\alpha_{i}=0,(i=1,2)$ and $\beta_{j}=0,(j=1,2,3)$, the model reduces to classical Navier stokes viscous fluid model [12].

Let the velocity field for the fluid flow be given in vector field. We assume the flow to be in the positive $\mathrm{z}$-direction. This implies that the pressure gradient must be negative and the following must hold.

1) The velocity field is independent of the coordinate $z$ and $\theta$. That is, $\bar{u}_{r r}, \bar{u}_{\theta \theta}, \bar{u}_{z z}, \bar{u}_{r \theta}, \bar{u}_{r z}, \bar{u}_{\theta r}, \bar{u}_{\theta z}, \bar{u}_{z \theta}=0$.

2) The extra stress being the result of the velocity field, are also independent of the $z$ and $\theta$. That is, $\tau_{r r}, \tau_{\theta \theta}, \tau_{z z}, \tau_{r \theta}, \tau_{\theta r}=\tau_{\theta z}=0$.

3) The sheer stress $\tau_{\theta z}$ and $\tau_{z \theta}$ on planes through the axis of the pipe are zero due to symmetry. This implies that the velocity field and the shear stresses are functions of $r$ alone.

Thus, the constitutive equation of motion for a third grade fluid flow is

$$
\tau=\bar{\mu} \frac{\partial \bar{u}}{\partial \bar{r}}+\alpha_{1} \frac{\partial^{2} \bar{u}}{\partial \bar{t} \partial \bar{r}}+2 \beta_{3}\left(\frac{\partial \bar{u}}{\partial \bar{r}}\right)^{3}
$$

Since the pressure gradient is a function of $\bar{z}$ and $\bar{t}$ we assume

$$
-\frac{\partial}{\partial \bar{z}} \bar{p}=\bar{q}_{0}+A_{1} \cos \bar{\omega} \bar{t}, \bar{t} \geq 0
$$

The boundary conditions are given as follows.

$$
\begin{aligned}
& \bar{\tau}_{T} \text { is finite at } \bar{r}=0 \\
& \bar{u}_{T}=0 \text { at } \bar{r}=\bar{R}(\bar{z})
\end{aligned}
$$

The geometry of the stenosis is given in Figure 1 and is defined by

$$
\bar{R}(\bar{z})=\left\{\begin{array}{l}
\bar{R}_{0}-\left(\frac{\bar{\delta}_{E}}{2}\right)\left\{1+\cos \left[\frac{2 \pi}{\bar{l}_{0}}\left(\bar{z}-\bar{d}-\frac{\overline{l_{0}}}{2}\right)\right]\right\} \text { in } \bar{d} \leq \bar{z} \leq \bar{d}+\bar{l}_{0} \\
\bar{R}_{0} \quad \text { in the normal artery region }
\end{array}\right.
$$

where $\bar{\delta}_{E}$ is the height of the stenosis.

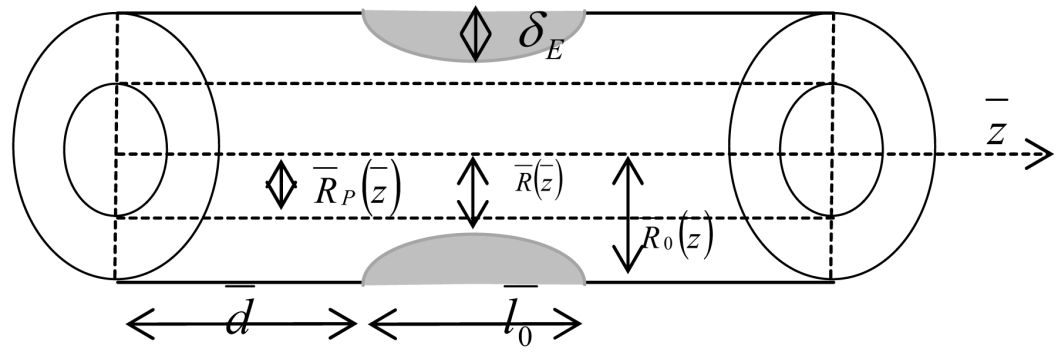

Figure 1. Geometry of the stenosed artery. 


\section{The Dimensional Analysis of the Equations}

The key to solving modern problems is mathematical modeling. This process involves keeping certain elements, neglecting some, and approximating yet others.

We present the following non-dimensional variables

$$
\left.\begin{array}{l}
z=\frac{\bar{z}}{\bar{R}_{0}}, r=\frac{\bar{r}}{\bar{R}_{0}}, R(z)=\frac{\bar{R}(\bar{z})}{\bar{R}_{0}}, q(z)=\frac{\bar{q}(\bar{z})}{\bar{R}_{0}}, \delta_{E}=\frac{\overline{\delta_{E}}}{\bar{R}_{0}}, t=\bar{\omega} \bar{t}, \alpha_{T}^{2}=\frac{\overline{R_{0}^{2}} \bar{\omega} \bar{\rho}_{T}}{\bar{\mu}_{T}}, \\
\ell=\frac{\bar{A}_{1}}{\bar{q}_{0}}, u_{T}=\frac{\bar{\mu}_{T}}{\bar{q}_{0} \bar{R}_{0}^{2} / 4 \bar{\mu}_{T}}, R_{p}=\frac{\bar{R}_{p}}{\bar{R}_{0}}, \tau_{T}=\frac{\bar{\tau}_{T}}{\bar{q}_{0} \bar{R}_{0} / 2}, \tau_{N}=\frac{\bar{\tau}_{N}}{\bar{q}_{0} \bar{R}_{0} / 2}, H=\frac{\bar{H}}{\bar{q}_{0}}
\end{array}\right\}
$$

Thus, Substituting (14) into (2), (9), (10) and (13) gives

$$
\begin{gathered}
\frac{\alpha_{T}^{2}}{4} \frac{\partial}{\partial t} u_{T}=(1+\ell \cos (t))-\frac{1}{2 r} \frac{\partial}{\partial r}\left(r \tau_{T}\right)+F \frac{\partial}{\partial z} H \\
\tau_{T}=\frac{1}{2} \frac{\partial u_{T}}{\partial r}+\alpha^{2} \Omega_{1} \frac{\partial^{2} u_{T}}{\partial t \partial r}+\alpha^{2} \Lambda_{1}\left(\frac{\partial u_{T}}{\partial r}\right)^{3}, \\
\text { if } 0 \leq r \leq R
\end{gathered}
$$

and the boundary conditions becomes

$$
\begin{gathered}
\tau \text { is finite and } \frac{\partial u}{\partial r}=0 \\
u_{T}=0 \text { at } r=R \\
R(z)=\left\{\begin{array}{l}
1-\left(\frac{\delta_{E}}{2}\right)\left\{1+\cos \left[\frac{2 \pi}{l_{0}}\left(z-d-\frac{l_{0}}{2}\right)\right]\right\} \text { in } d \leq z \leq d+l_{0} \\
1 \quad \text { in the normal artery region. }
\end{array}\right.
\end{gathered}
$$

\section{Solution of the Model Using Regular Perturbation Method}

Let us expand the flow characteristics in (15) and (16) in the perturbation series below taking $\alpha_{T}^{2}$ as the perturbation parameter.

$$
\begin{aligned}
& \tau_{T}=\tau_{T 0}+\alpha_{T}^{2} \tau_{T 1}+\cdots \\
& u_{T}=u_{T 0}+\alpha_{T}^{2} u_{T 1}+\cdots
\end{aligned}
$$

If we equate the coefficients of $\alpha^{2}$ to have;

$$
\begin{gathered}
\alpha_{T}^{0}: \frac{2}{r} \frac{\partial}{\partial r}\left[r\left(\tau_{T 0}\right)\right]=4(1+\ell \cos (t))+F \frac{\partial}{\partial z} H \\
\tau_{T 0}=\frac{1}{2} \frac{\partial}{\partial r}\left(u_{T 0}\right) \\
\alpha_{T}^{2}:-\frac{2}{r} \frac{\partial}{\partial r}\left[r\left(\tau_{T 1}\right)\right]=\frac{\partial}{\partial t}\left(u_{T 0}\right) \\
\frac{1}{2} \frac{\partial}{\partial r}\left(u_{T 1}\right)+\Omega_{1} \frac{\partial^{2}}{\partial t \partial r}\left(u_{T 0}\right)+\Lambda_{1}\left(\frac{\partial}{\partial r}\left(u_{T 0}\right)\right)^{3}=\tau_{T 1}
\end{gathered}
$$

Thus, the solution set is given by 


$$
\left.\begin{array}{c}
\tau_{T}=\tau_{T 0}+\alpha_{T}^{2} \tau_{T 1}+\cdots \\
u_{T}=u_{T 0}+\alpha_{T}^{2} u_{T 1}+\cdots
\end{array}\right\}
$$

The volume flow rate is given by

$$
\begin{gathered}
Q=2 \pi \int_{0}^{R(z)} u_{T}(r, t) r \mathrm{~d} r \\
Q=2 \pi\left(-\frac{1}{4}(1+\ell \cos (t))\left(R^{4}\right)-\frac{1}{16} F \frac{\partial}{\partial z} H\left(R^{4}\right)+\frac{1}{32} \Lambda_{1} \alpha_{T}^{2}\left(F \frac{\partial}{\partial z} H\right)^{3}\left(R^{6}\right)\right. \\
-\frac{1}{2} \Omega_{1} \alpha_{T}^{2} \ell \sin (t)\left(R^{4}\right)+\frac{1}{4} \Lambda_{1} \alpha_{T}^{2}(1+\ell \cos (t))\left(F \frac{\partial}{\partial z} H\right)^{2}\left(R^{6}\right) \\
+\frac{4}{3} \Lambda_{1} \alpha_{T}^{2}(1+\ell \cos (t))^{3}\left(R^{6}\right)-\frac{1}{24} \alpha_{T}^{2} \ell \sin (t)\left(R^{6}\right) \\
\left.+\frac{2}{3} \Lambda_{1} \alpha_{T}^{2}(1+\ell \cos (t))^{2}\left(F \frac{\partial}{\partial z} H\right)\left(R^{6}\right)\right)
\end{gathered}
$$

The resistance to the flow is given by

$$
\begin{aligned}
\Lambda=\frac{-\frac{\partial}{\partial z}(P)}{Q} \\
\Lambda=\frac{(1+\ell \cos (t))}{2 \pi\left(-\frac{1}{4}(1+\ell \cos (t))\left(R^{4}\right)-\frac{1}{16} F \frac{\partial}{\partial z} H\left(R^{4}\right)\right.} \\
+\frac{1}{32} \Lambda_{1} \alpha_{T}^{2}\left(F \frac{\partial}{\partial z} H\right)^{3}\left(R^{6}\right)-\frac{1}{2} \Omega_{1} \alpha_{T}^{2} \ell \sin (t)\left(R^{4}\right) \\
+\frac{1}{4} \Lambda_{1} \alpha_{T}^{2}(1+\ell \cos (t))\left(F \frac{\partial}{\partial z} H\right)^{2}\left(R^{6}\right) \\
+\frac{4}{3} \Lambda_{1} \alpha_{T}^{2}(1+\ell \cos (t))^{3}\left(R^{6}\right)-\frac{1}{24} \alpha_{T}^{2} \ell \sin (t)\left(R^{6}\right) \\
\left.+\frac{2}{3} \Lambda_{1} \alpha_{T}^{2}(1+\ell \cos (t))^{2}\left(F \frac{\partial}{\partial z} H\right)\left(R^{6}\right)\right)
\end{aligned}
$$

The wall shear stress is given by

$$
\tau_{w}=\left.\tau_{T}\right|_{r=R}
$$




$$
\tau_{w}=(1+\ell \cos (t))(R)+\frac{1}{4} F \frac{\partial}{\partial z} H(R)-\frac{1}{8} \alpha_{T}^{2} \ell \sin (t)\left(R^{4}\right)
$$

\section{Results and Discussion}

We present this paper with the view to analyze the effect of magnetic field on pulsatile third grade blood flow in a stenosed artery. We have used the following parameter values; 0.2 is used for the dimensionless amplitude $\ell$. The Womersley numbers $\alpha_{T}$ is taken as 1.2. The Womersley number denotes the ratio of unsteady inertial forces to viscous forces in the flow. It ranges from as large as about 20 in the aorta, significantly greater than 1 in all large arteries, to as small as $10^{-3}$ in the capillaries [13]. 0.15 is used for $\delta_{T}$.

Figure 2 depicts the variation of the total volumetric flow rate in the artery geometry along the axial direction for different values of magnetic field intensity $H$, with $d=2, \ell=0.2, \ell=0.2, l=1, \delta_{T}=0.15$. It has been observed that, the total volume flow rate increases as the magnetic field intensity increases. However, this relation is for the volume flow rate along the axial direction before the location of the stenosis. Figure 3 shows that for the fluid model in the axial direction for the length of the arteries under study with stenosis, the volume flow rate possesses a periodic relation. Thus, it increases with increase in magnetic field intensity $H$. Figure 4 shows that for a constant value of magnetic field intensity of about 3 , the volume flow rate possesses a periodic relation. Thus, it increases with increase in shear thinning and reduces with increase in shear thickening as suggested by Figure $5 \&$ Figure 7 . Figure 6 \& Figure 7 are however relations just before the stenosis position with increasing shear thinning

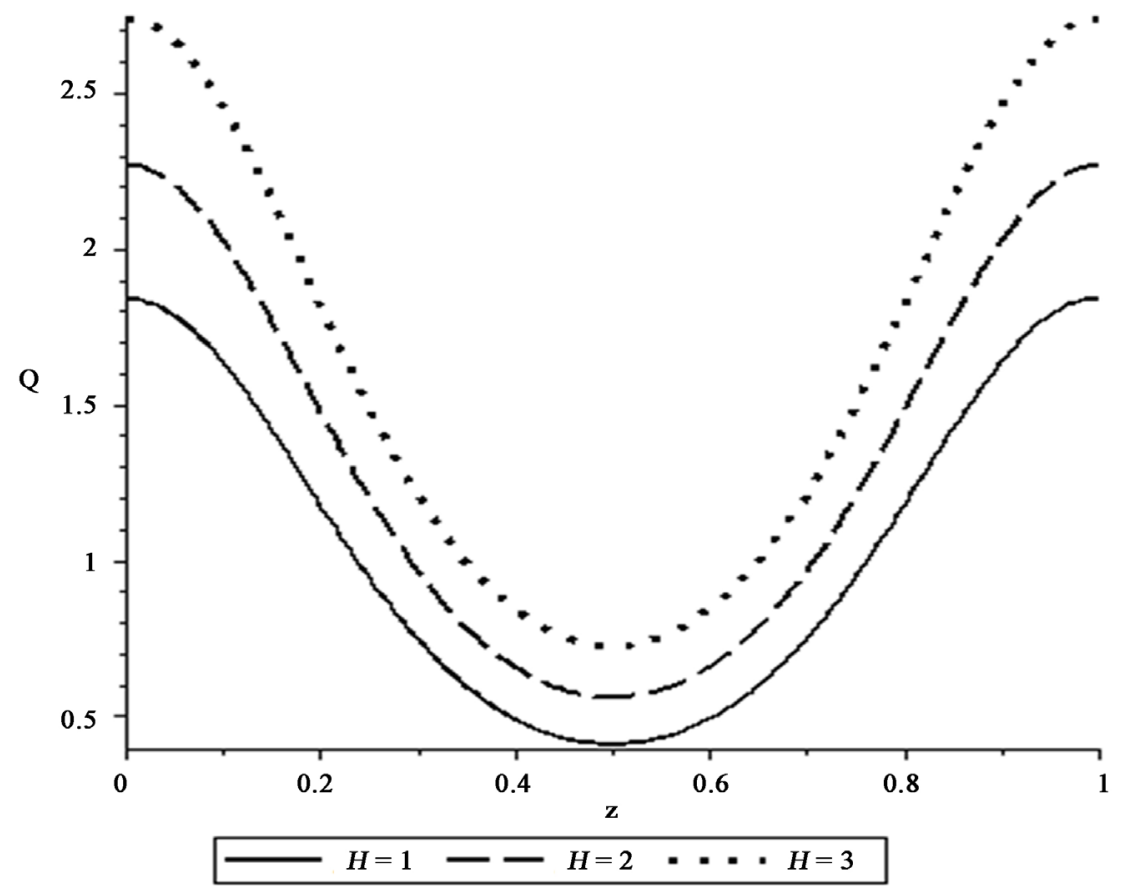

Figure 2. Variation of the volumetric flow rate with different values of magnetic field intensity in the axial direction before the stenosis position. 


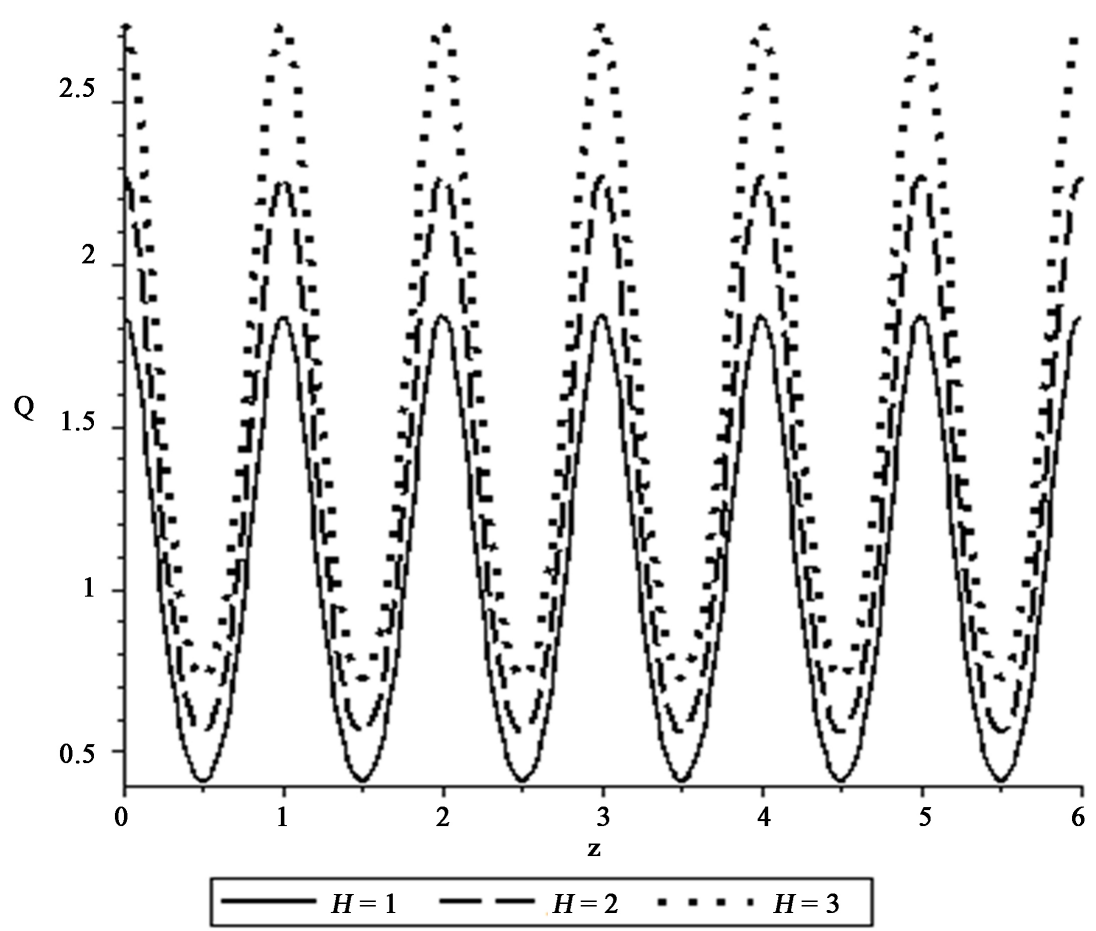

Figure 3. Variation of the volumetric flow rate with different values of magnetic field intensity in the axial direction after the stenosis position.

and thickening respectively. Figures 8-10 show the variation of the volume flow rate with time at different values of the shear thinning, shear thickening and magnetic field intensity $H$. The graphs shift away from the origin when the shear thinning and the magnetic field intensity increases and shift closer to the origin as the shear thickening increase. This shows that the volume flow rate increases with increase in shear thinning and magnetic field but reduces over time, the volume flow rate hits the zero bar after some time, implying a possible case of occlusion. This result is in agreement with the laboratory results of the work of [14].

Figures 11-13 show variation of velocity profile of the third grade fluid along the radial distance for different values of shear thickening, shear thinning and magnetic field $H$, with $d=2, \ell=0.2, \ell=0.2, \quad l=1, \delta_{T}=0.15$. The velocity curve shift away from the origin when the shear thinning \& magnetic field increase (Figure 12 \& Figure 13), and shift to the origin when the shear thickening increases. Thus, this shows that the velocity increases with increase in shear thinning \& magnetic field and reduce with increase in shear thickening.

Figures 14-16 show the variation of the total resistance to the volumetric flow rate in the artery geometry along the axial direction for different values of shear thickening, shear thinning and magnetic field intensity $H$, with $d=2, \ell=0.2$, $\ell=0.2, l=1, \delta_{T}=0.15$. We observed that increasing shear thickening offers high resistance to the volume flow rate (Figure 14), this will result to more pressure forces exerting on the heart which serves as the only pressure 


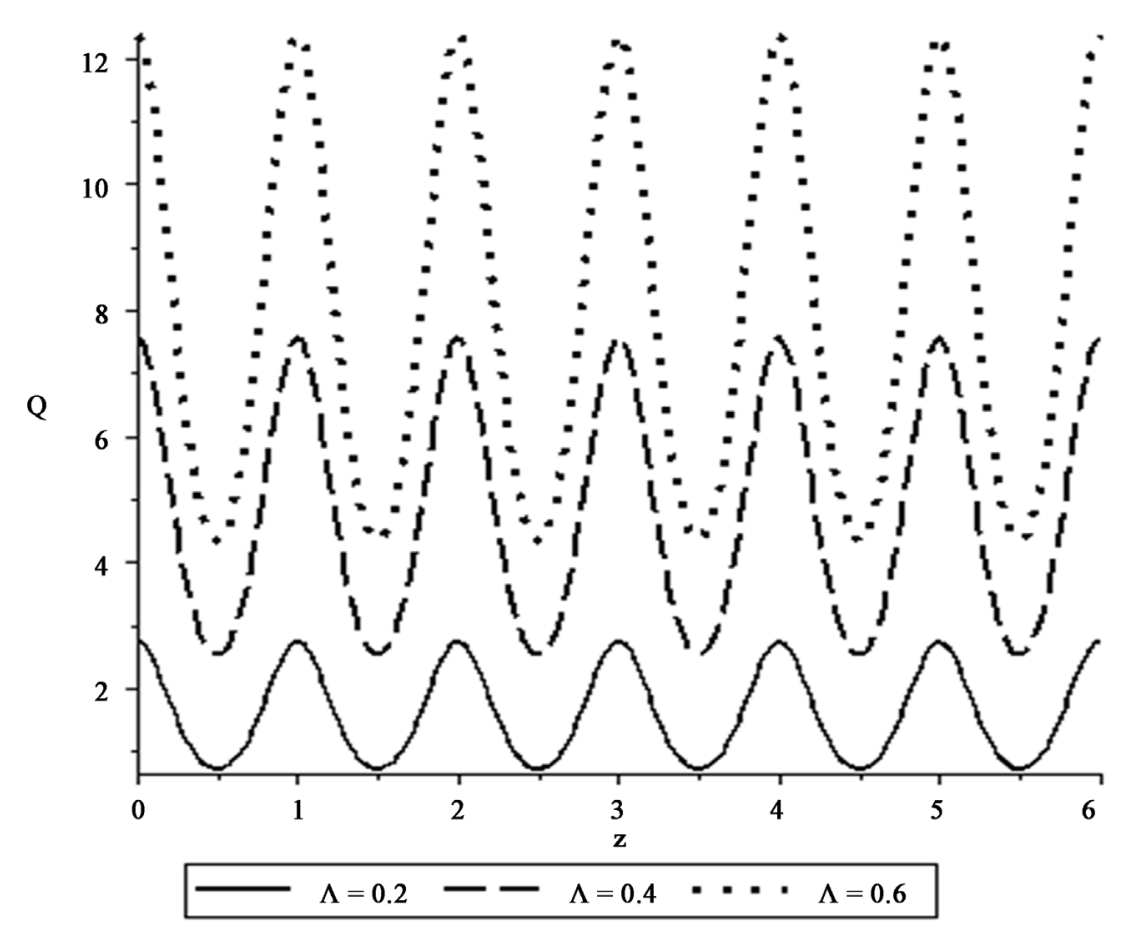

Figure 4. Variation of the volumetric flow rate with different values of shear thinning in the axial direction after the stenosis' position.

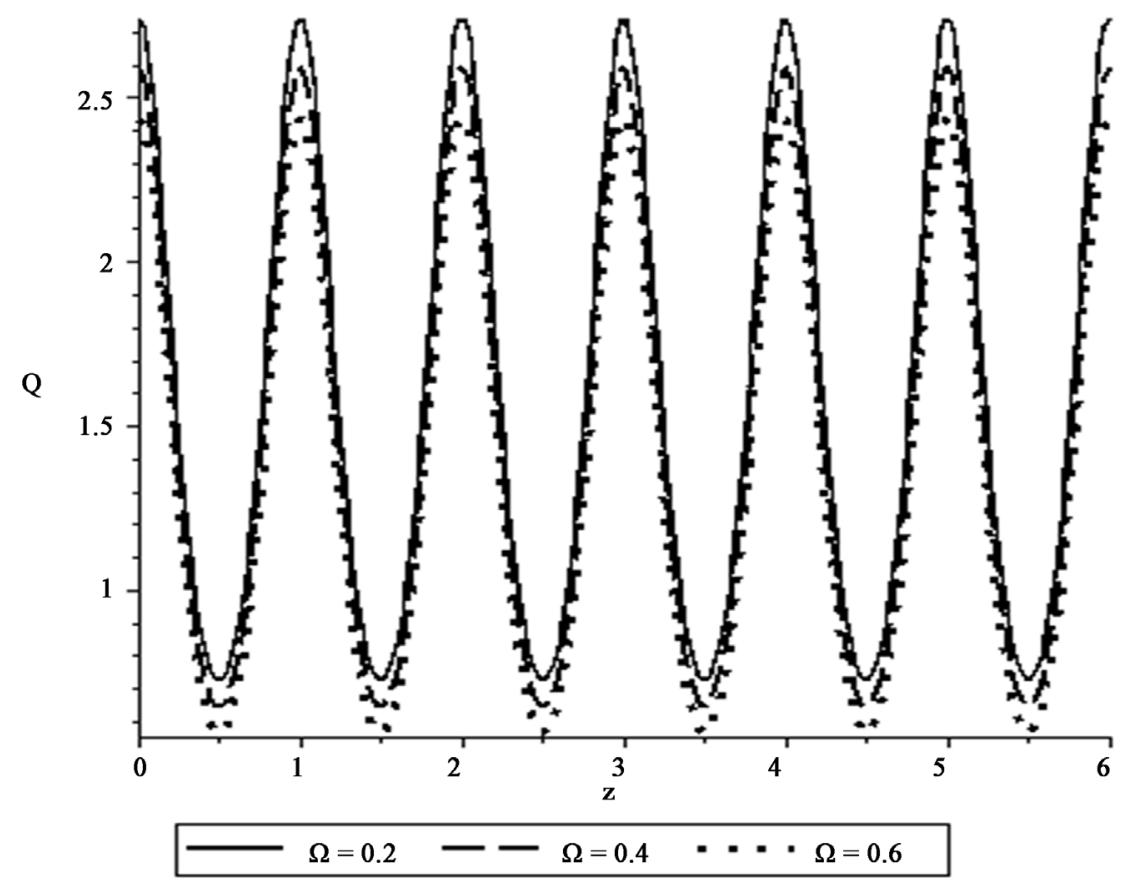

Figure 5. Variation of the volumetric flow rate with different values of shear thickening in the axial direction after the stenosis' position.

source to drive the flow of blood thereby weakening the muscular walls of the heart resulting to myocardial infarction (in which part of the heart muscle dies) or cardiac arrest (in which blood flow stops altogether). In [15], the author 


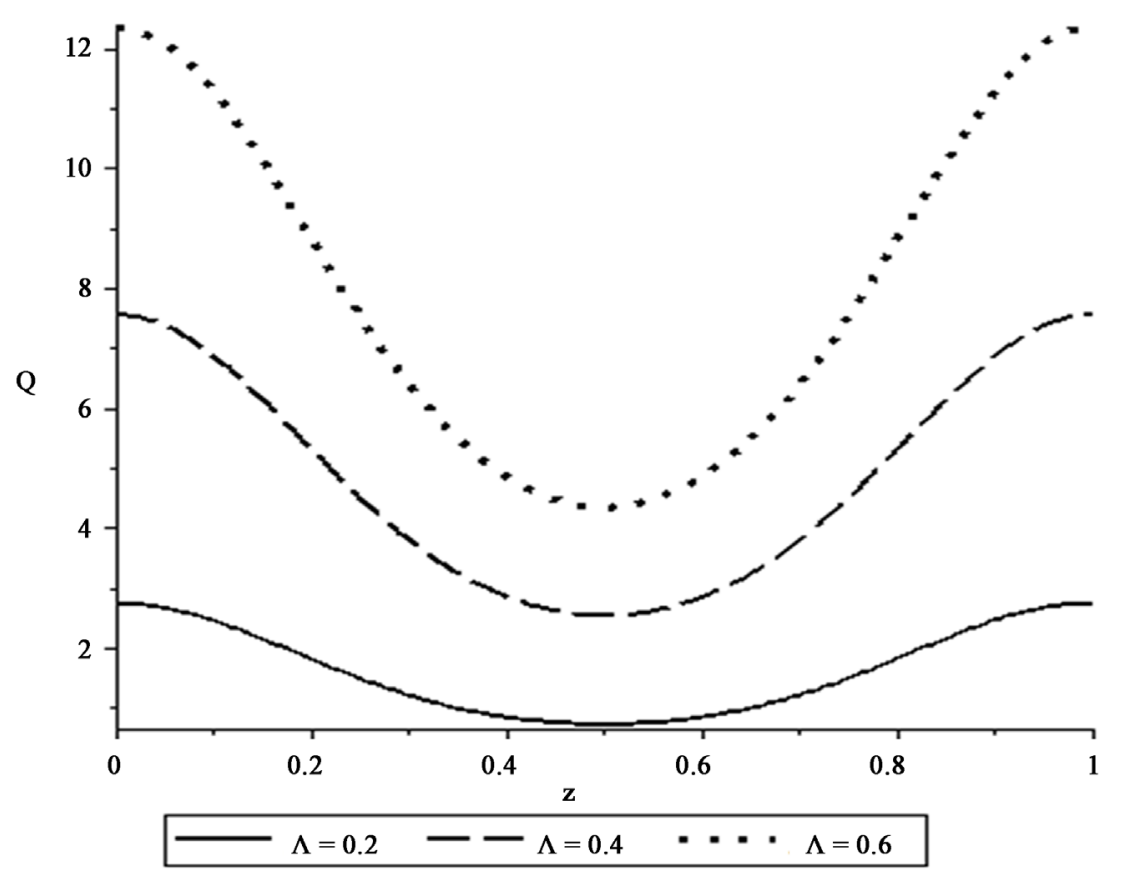

Figure 6. Variation of the volumetric flow rate with different values of shear thinning in the axial direction before the stenosis' position.

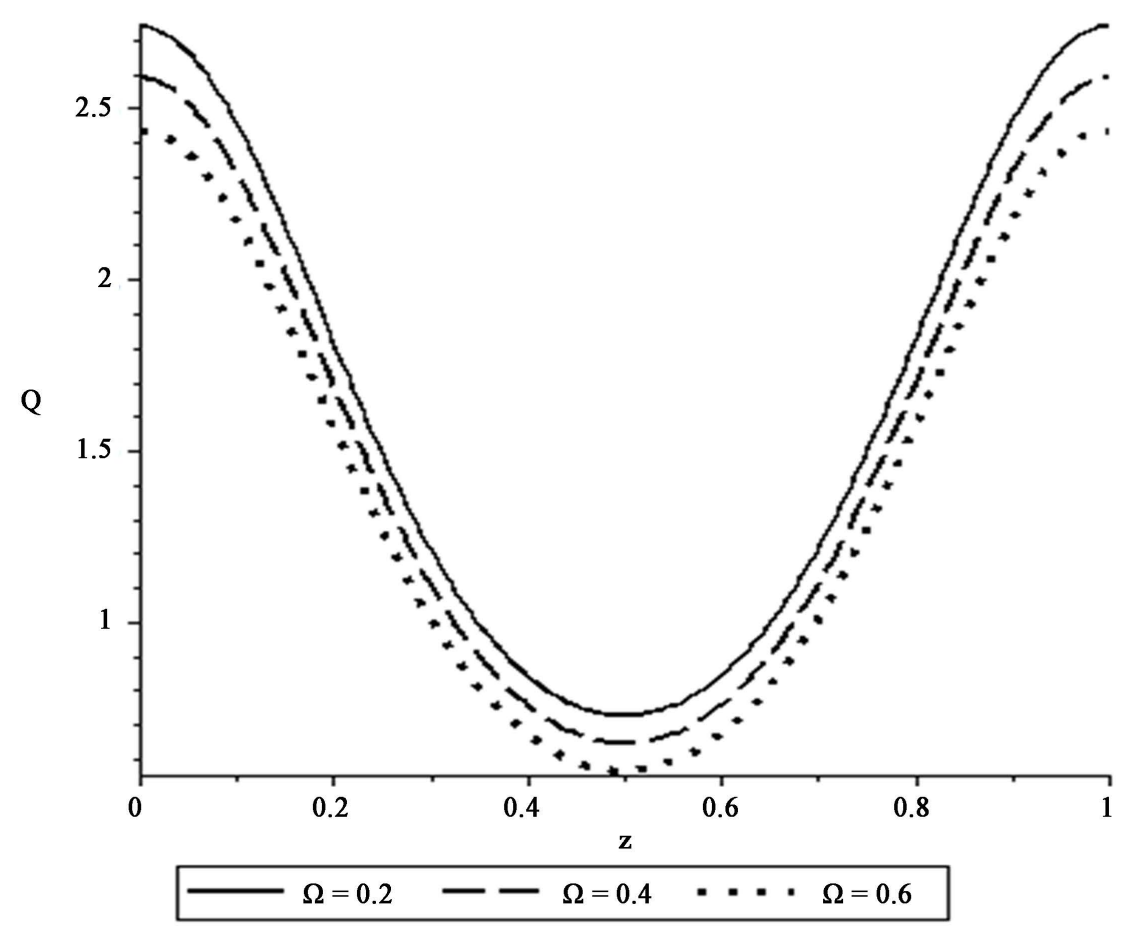

Figure 7. Variation of the volumetric flow rate with different values of shear thickening in the axial direction before the stenosis' position.

opines that this is the cause of the sudden break down of the entire circulatory system leading to death in recent time. Increasing shear thinning reduces the resistance to flow (Figure 15) and the resistance to flow decreases as the magnetic 


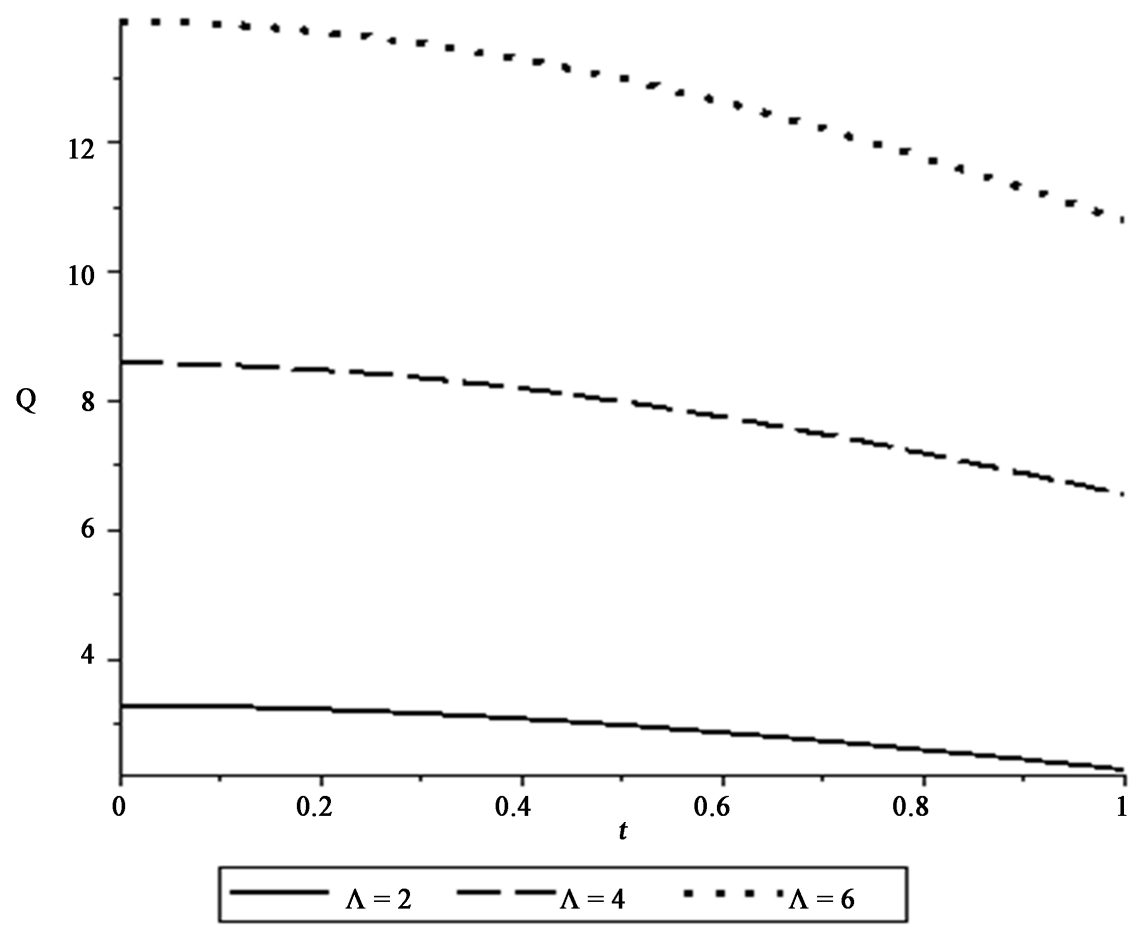

Figure 8. Variation of the volumetric flow rate with increasing shear thinning in the axial direction over time.

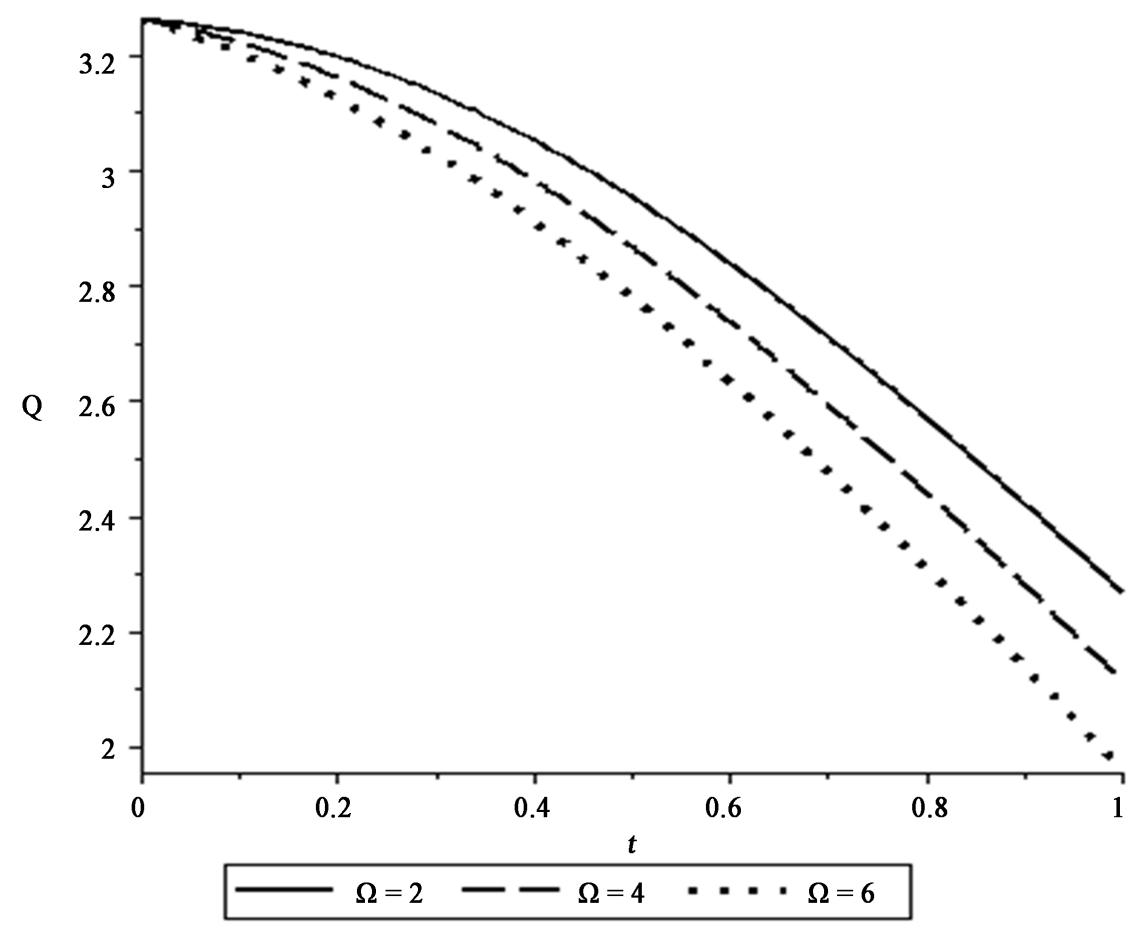

Figure 9. Variation of the volumetric flow rate with increasing shear thickening in the axial direction over time.

field intensity increases (Figure 16). That is, increasing magnetic field strength offers low resistance to the flow. 


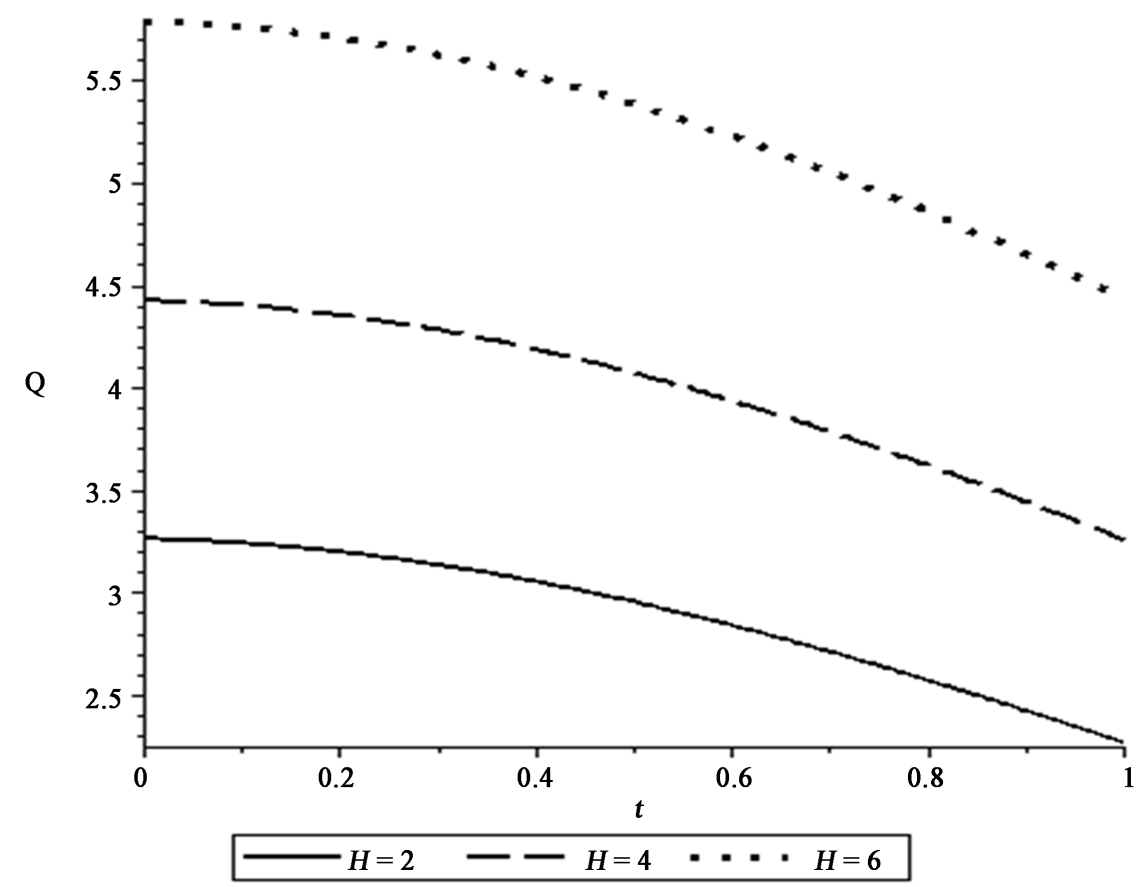

Figure 10. Variation of the volumetric flow rate with increasing magnetic field intensity in the axial direction over time.

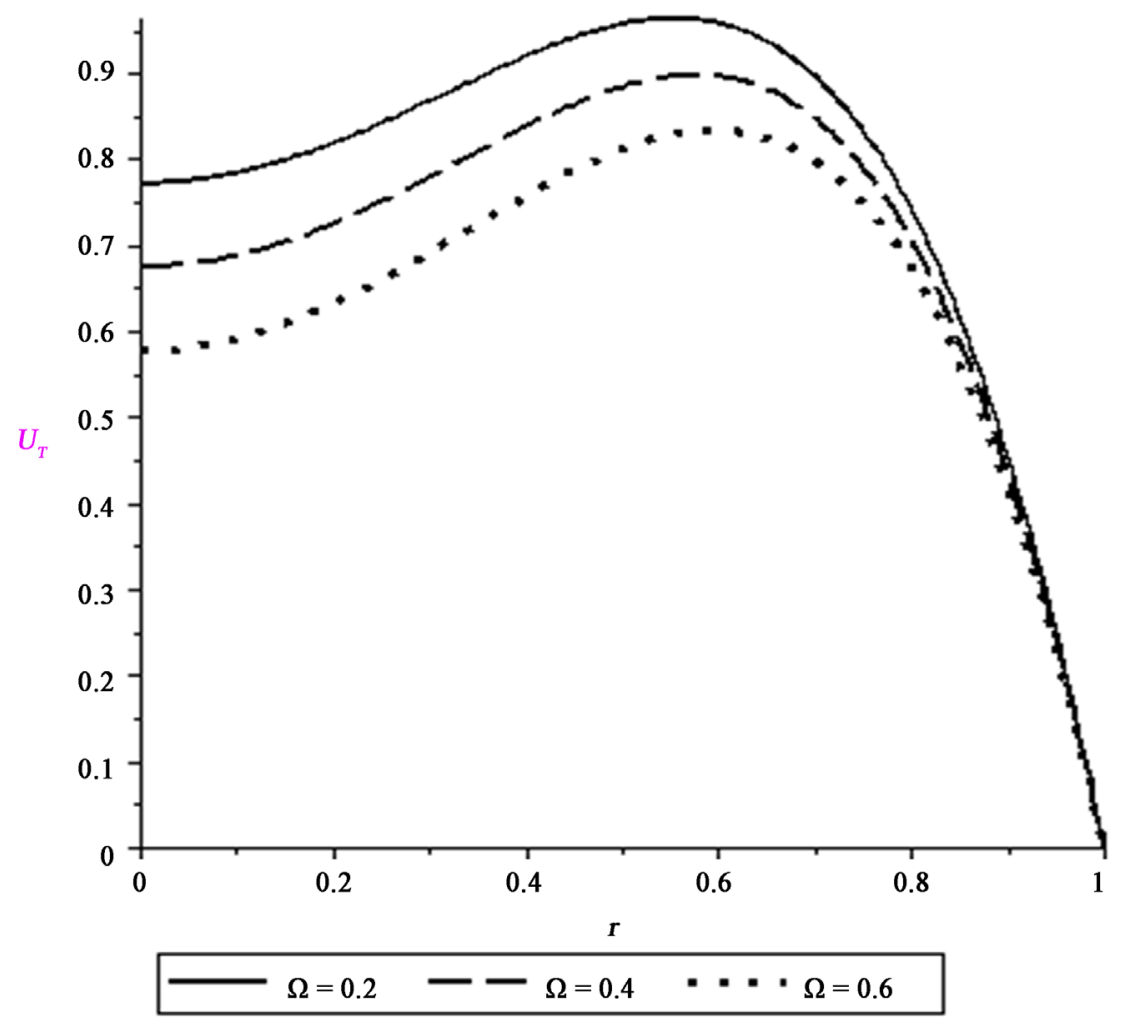

Figure 11. The velocity profile with increasing shear thickening.

Figure 16-18 show the shear stress profile for different values of magnetic field intensity $H$, with $d=2, \ell=0.2, \ell=0.2, l=1, \delta_{T}=0.15$. It has been 


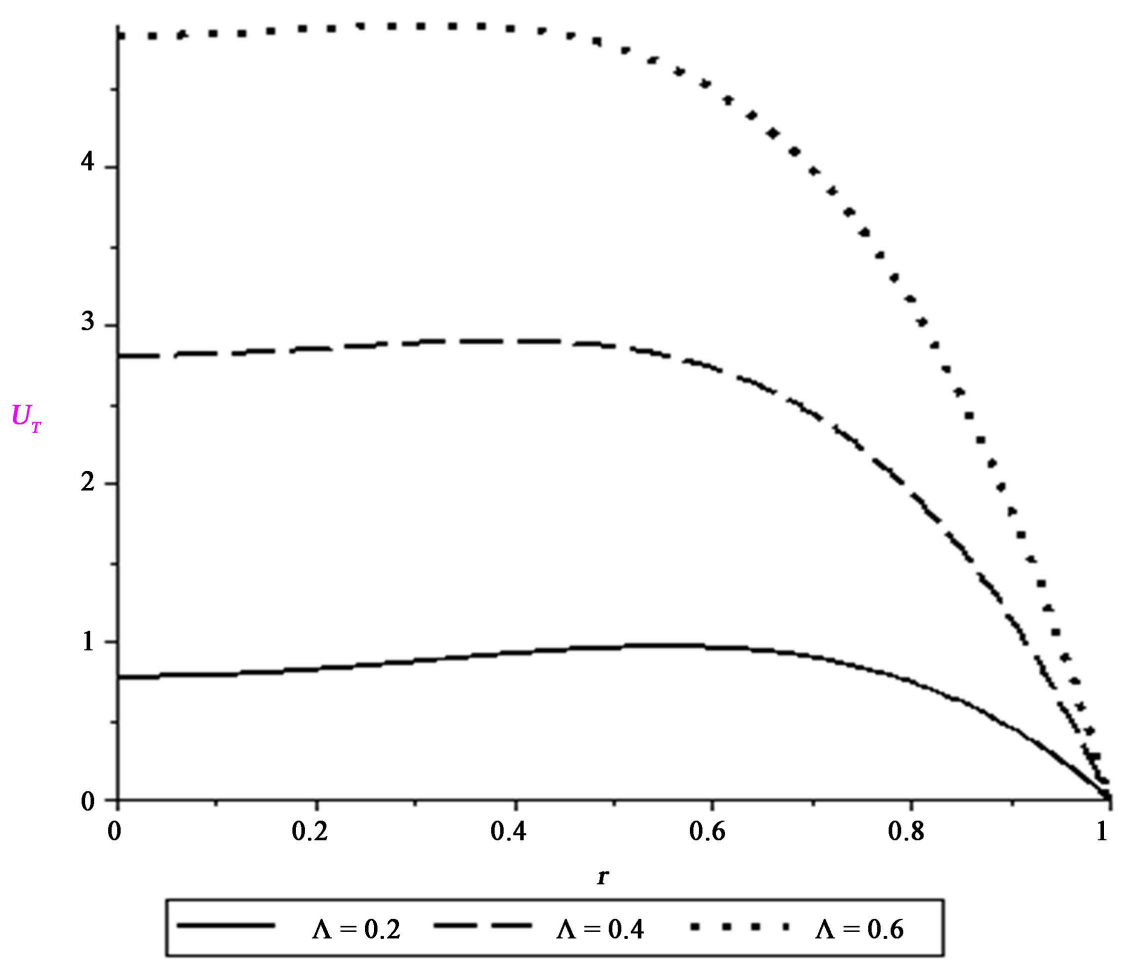

Figure 12. The velocity profile with increasing shear thinning.

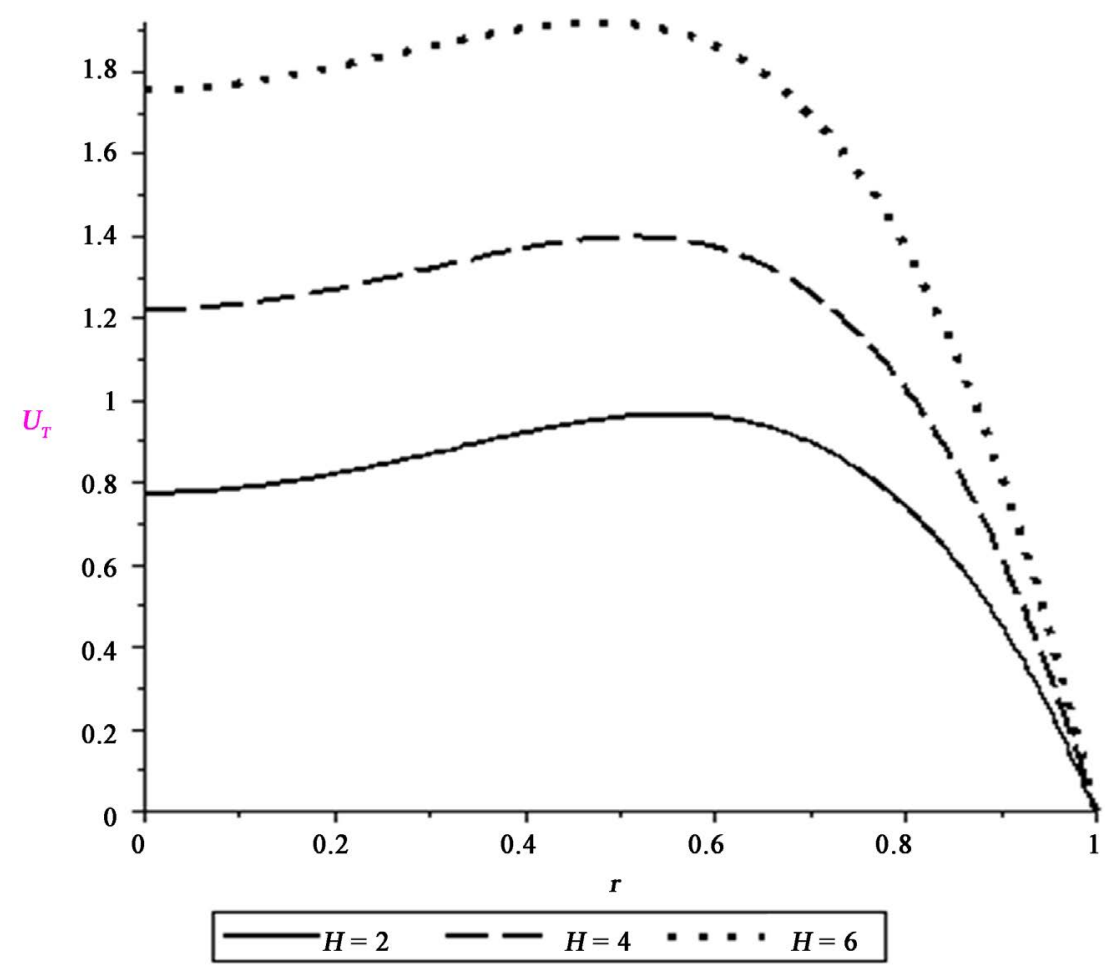

Figure 13 . The velocity profile with increasing shear thickening.

observed that the wall shear stress behave similar to the volume flow rate. The graphs shift away from the origin as the magnetic field increases with a periodic 


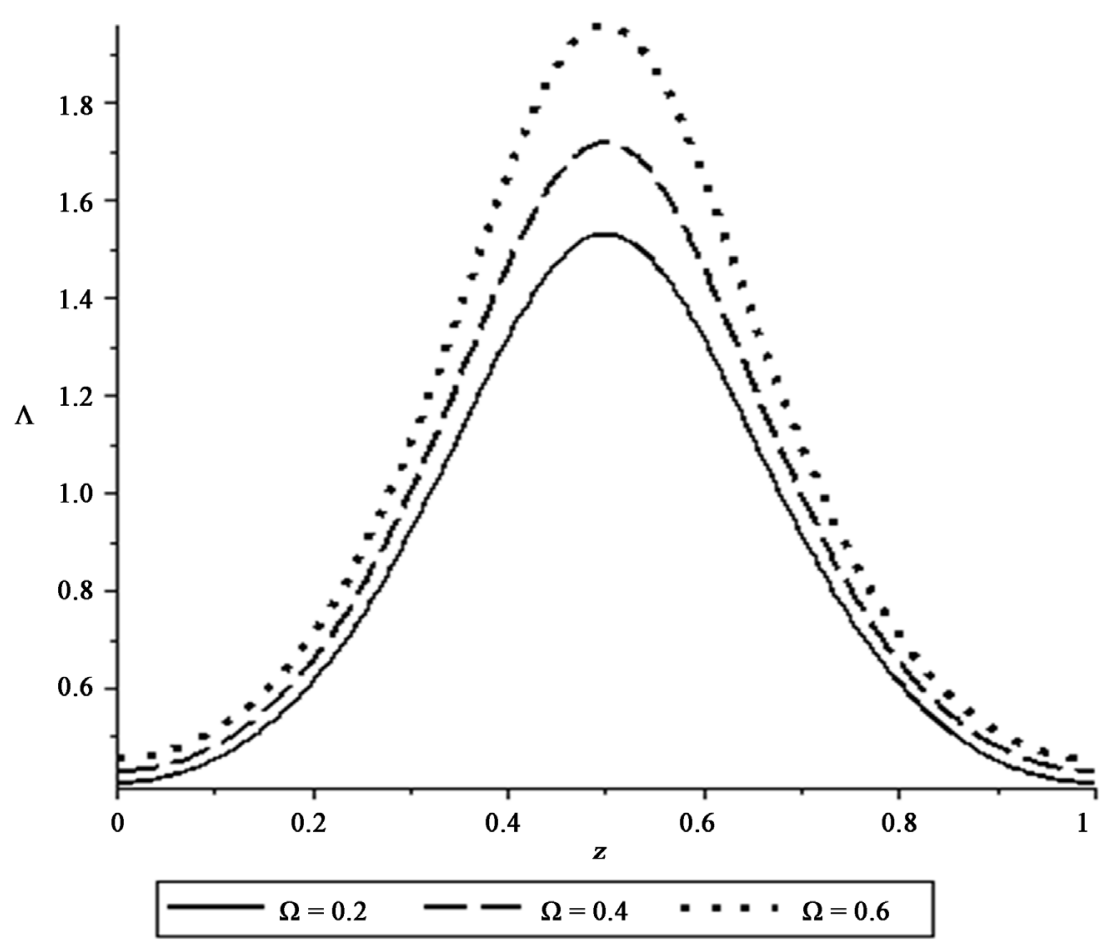

Figure 14. The resistances to flow with increasing shear thickening.

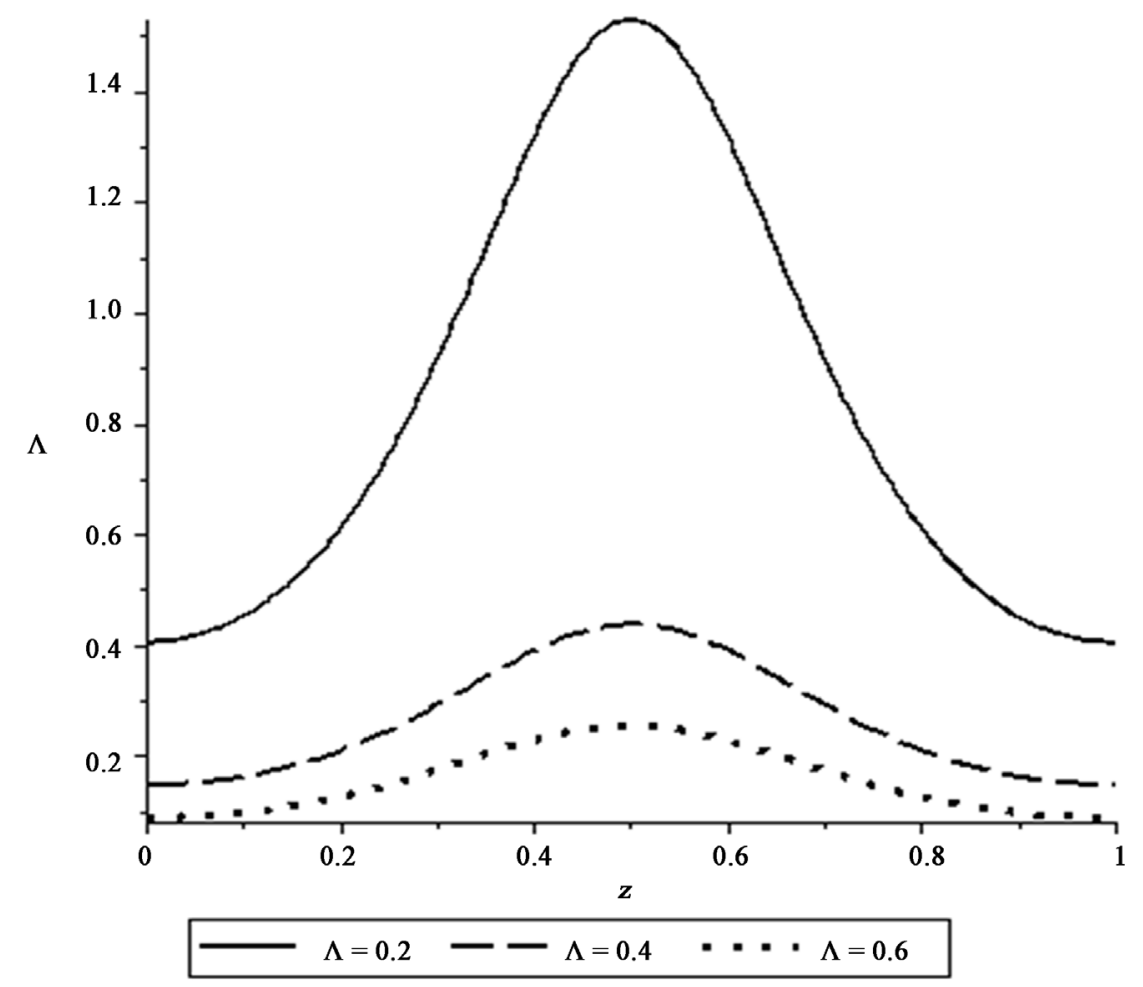

Figure 15. The resistances to flow with increasing shear thinning.

relation in Figure 16, which explains the effect of the stenosis in the artery along the direction of flow. 


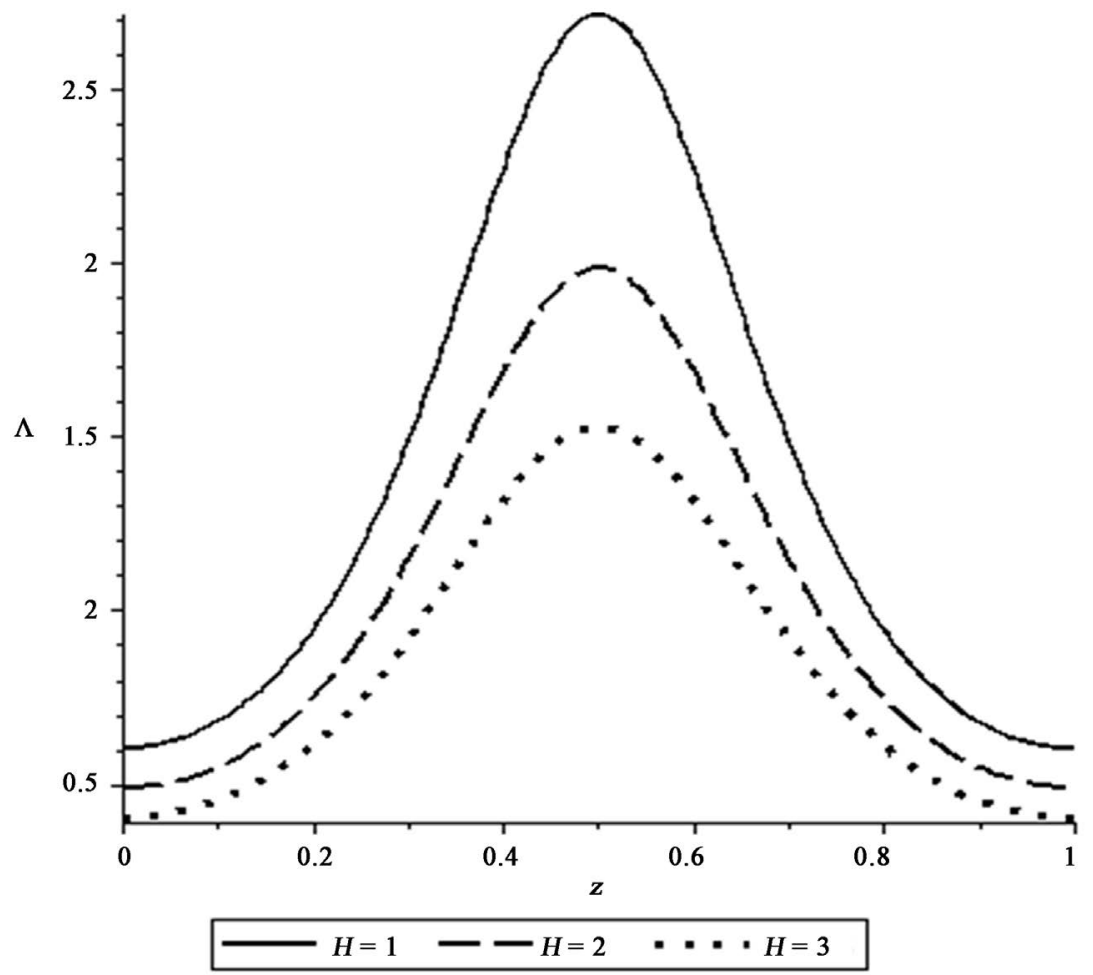

Figure 16. The resistance to flow with increasing shear thickening.

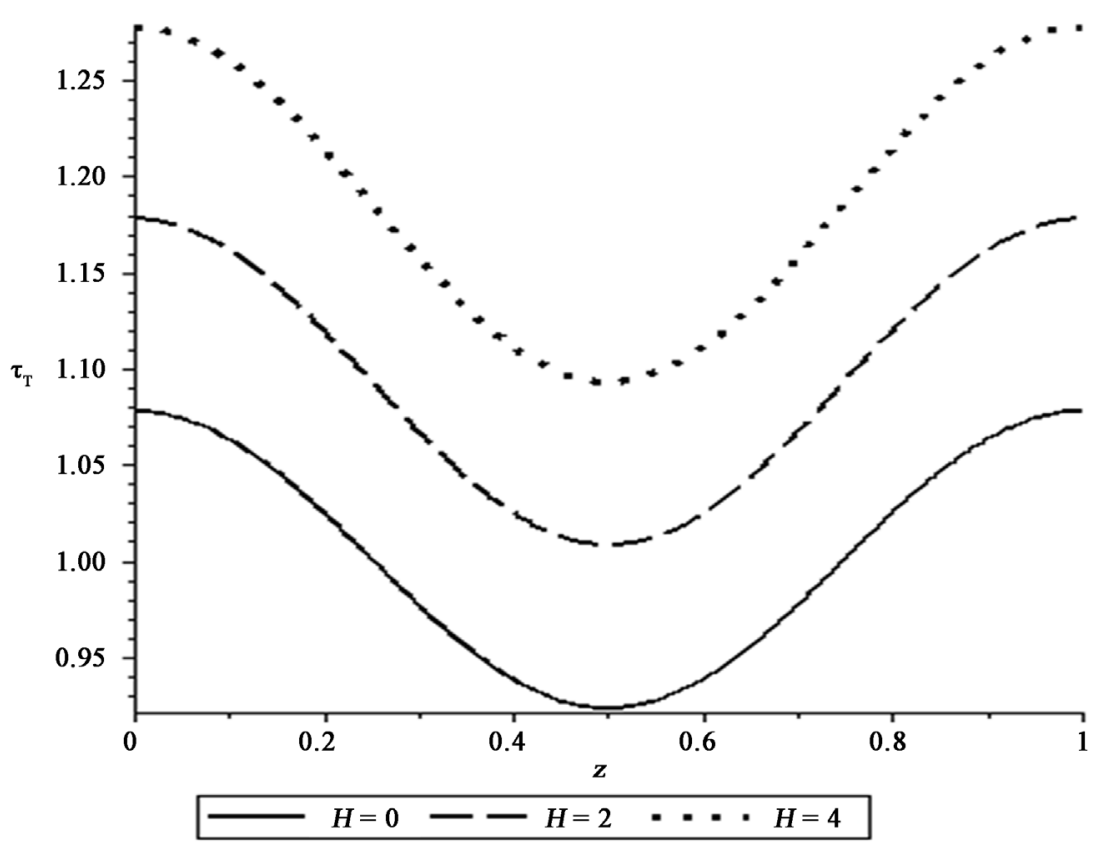

Figure 17. Theshear stress with increasing magnetic field intensity in the axial direction.

\section{Conclusion}

Any fluid that does not obey Newton's Law of Viscosity is a non-Newtonian fluid. The viscosity of such fluids is mostly dependent on their shear rate or the shear rate history. In fluid mechanics, the fluid property called viscosity is used 


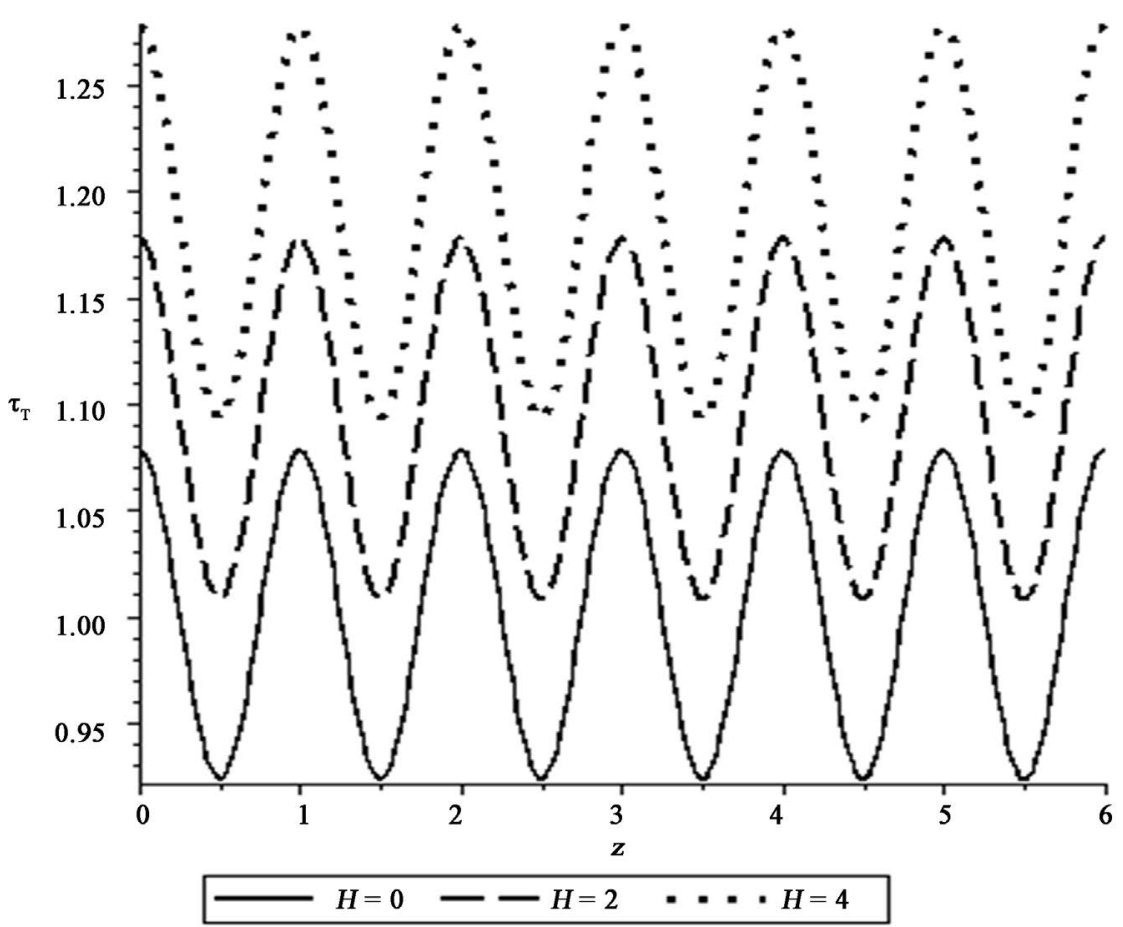

Figure 18. Theshear stress with increasing magnetic field intensity in the axial direction after the stenosis' position.

to characterize the shear properties of a fluid, which is inadequate to describe non-Newtonian fluids. Non-Newtonian fluids are best studied through several other rheological properties that relate stress and strain rate tensors under many different flow conditions. An example of a non-Newtonian fluid with the shear thinning and thickening properties is blood which we considered as third grade fluid. This application is highly valued for its importance in understanding flows in defective arteries within the body, as it allows us to relate the magnetic field and blood to further understand the flow characteristics of blood through a stenosed artery.

\section{References}

[1] Buchanan Jr., J.R., Kleinstreuer, C. and Corner, J.K. (2000) Rheological Effects on Pulsatile Hemodynamics in a Stenosed Tube. Journal of Computers \& Fluids, 29, 695-724. https://doi.org/10.1016/S0045-7930(99)00019-5

[2] Swift, M.R. and Weinstein, B.M. (2009) Arterial-Venous Specification during Development. Circulation Research, 104, 576-588. https://doi.org/10.1161/CIRCRESAHA.108.188805

[3] Miller, J.D. (2013) Cardiovascular Calcification: Orbicular Origins. Nature Materials, 12, 476-478. https://doi.org/10.1038/nmat3663

[4] Zeb, M., Islam, S., Siddiqui, A.M. and Haroon, T. (2013) Analysis of Third-Grade Fluid in Helical Screw Rheometer. Journal of Applied Mathematics, 2013, 1-11. https://doi.org/10.1155/2013/620238

[5] Hayat, T., Anum, S. and Alsaedi, A. (2015) MHD Axisymetric Flow of Third Grade Fluid by a Stretching Cylinder. Alexandria Engineering Journal, 54, 205-212. 
https://doi.org/10.1016/j.aej.2015.03.013

[6] Maurino, R.M. (1991) From Thales to Lauterbur, or from Lodestone to MR Imaging: Magnetism and Medicine. Radiology, 180, 593-612.

https://doi.org/10.1148/radiology.180.3.1871268

[7] Jerabek, J. and Pawluk, W. (1998) Magnetic Therapy in Eastern Europe: A Review of 30 Years of Research. 2nd Edition, Paperback Publishers, 320.

[8] Iwasaka, M. and Ueno, S. (1998) Structure of Water Molecules under 14 Tesla Magnetic Field. Journal of Applied Physics, 83, 87-95.

https://doi.org/10.1063/1.367737

[9] Kai-Tai, C. and Cheng, W. (2006) The Effect of an External Magnetic Field on the Structure of Liquid Water Using Molecular Dynamics Simulation. Journal of Applied Physics, 100, 1-6.

[10] Tzirtzilakis, E.E. (2005) A Mathematical Model for Blood Flow in Magnetic Field. Physics of Fluids, 17, 1-15. https://doi.org/10.1063/1.1978807

[11] Alia, A.K., Baidaa, T.S. and Alauldeen, M.Z. (2016) Influence of Magnetic Field on Blood Viscosity. Journal of Advances in Environmental Biology, 10, 107-110.

[12] Fernando, C. (2008) Axisymmetric Motion of a Generalized Rivlin-Ericksen Fluids with Shear-Dependent Normal Stress Coefficients. International Journal of Mathematical Models and Methods in Applied Sciences, 2, 168-175.

[13] Pijush, K.K. and Ira, M.C. (2008) Fluid Mechanics. 4th Edition, Academic Press, Cambridge, Massachusetts, 782.

[14] Tao, R. and Huang, K. (2011) Reducing Blood Viscosity Using Magnetic Field. Physical Review E, 84, 001-015. https://doi.org/10.1103/PhysRevE.84.011905

[15] Eyal, H. (2012) The Cardiac Care Unit Survival Guide. Lippincott Williams \& Wilkins, Philadelphia, 98. 


\section{Nomenclatures}

$\bar{R}(\bar{z})$ - Radius of the stenosed artery

$\bar{\delta}_{E}$ - Maximum height of the stenosis

$\overline{I_{0}}$ - The length of stenosis.

$\bar{d}$ - Location of the stenosis.

$\bar{z}$ - The axial distance.

$\bar{r}$ - The radial distance.

$\bar{\tau}_{T}$ - The shear stress for the Third grade fluid.

$\bar{\rho}_{T}$ - The density of the Third grade fluid.

$\bar{p}$ - The pressure force.

$\bar{\mu}_{T}$ - Viscosity of the Third grade fluid.

$\bar{U}_{T}$ - Velosity of the Third grade fluid.

$M$ - Magnetisation of the fluid.

$\mu_{0}$ - The magnetic permeability.

$\bar{H}$ - The magnetic field intensity.

$\bar{R}_{p}$ - The plug core radius. 